\title{
CALAGEM E GESSAGEM EM SISTEMA DE SEMEADURA DIRETA DE SOJA NO NOROESTE PAULISTA
}

\author{
BORGES, Wander Luis Barbosa ${ }^{1}$; TOKUDA, Flávio Sueo ${ }^{2}$; HIPÓLITO, Jorge Luiz ${ }^{3}$; SOUZA, Isabela \\ Malaquias Dalto de ${ }^{4}$; ANDREOTTI, Marcelo ${ }^{5}$; GASPARINO, Adriano Custódio ${ }^{6}$
}

ISSUE DOI: $10.3738 / 1982.2278 .3627$

\begin{abstract}
RESUMO: Sistemas de manejo conservacionistas que têm o intuito de evitar o desgaste ou a degradação do solo vêm sendo difundidos na agricultura. Entre esses sistemas está o sistema de semeadura direta. O presente trabalho foi realizado com o objetivo avaliar o efeito da calagem e da gessagem sobre a cultura da soja cultivada no sistema de semeadura direta na região Noroeste Paulista. O delineamento experimental utilizado foi em blocos casualizados com quatro tratamentos (T1 - calagem e gessagem para estabelecer que, respectivamente, o cálcio ocupe $70 \%$ da CTC na camada de 0-0,20 m e 60\% da CTCe na camada de 0,20-0,40 m; T2 - calagem e gessagem para estabelecer que o cálcio ocupe $52,5 \%$ da CTC na camada de 0-0,20 m e 45\% da CTCe na camada de 0,20-0,40 m; T3 - calagem e gessagem para estabelecer que o cálcio ocupe $35 \%$ da CTC na camada de $0-0,20 \mathrm{~m}$ e $30 \%$ da CTCe na camada de 0,20-0,40 m; T4 - tratamento padrão, sem aplicação de calcário e gesso agrícola) e quatro repetições. Os parâmetros avaliados na cultura da soja foram: altura de inserção da primeira vagem, altura de plantas, estande final ha ${ }^{-1}$, massa de cem grãos e produtividade de grãos ha ${ }^{-1}$. Os dados foram submetidos ao teste $\mathrm{F}$ e as médias foram comparadas pelo teste de Tukey $(\mathrm{p}<0,05)$. Constatou-se que o tratamento T2 apresentou maior altura de inserção da primeira vagem que o tratamento padrão. Para as demais características agronômicas avaliadas não houve diferença entre os tratamentos.
\end{abstract}

Palavras-chave: Glycine max (L.) Merrill. Corretivos. Características agronômicas.

\section{LIMING AND GYPSUM APPLICATION IN NO-TILLAGE OF SOYBEAN IN THE NORTHWEST REGION OF SÃO PAULO STATE, BRAZIL}

\begin{abstract}
SUMMARY: Conservation management systems that have the intention of avoiding soil erosion or degradation have been diffused in agriculture. Among these systems is the no-tillage system. The present work was carried out with the objective of evaluating the effect of limestone and agricultural gypsum application on the cultivation of soybean grown in the no-tillage system. The experimental design was a randomized block with four treatments (T1 limestone and agricultural gypsum application to establish that, respectively, calcium occupy $70 \%$ of the CTC in the 0-0.20 and $60 \%$ of the CTCe layer in the 0.20-0.40 m layer - T2 - limestone and agricultural gypsum application to establish that calcium occupies $52.5 \%$ of the CTC in the layer of $0-0,20 \mathrm{~m}$ and $45 \%$ of the CTCe in the layer of 0,20 $0,40 \mathrm{~m}$; T3 - limestone and agricultural gypsum to establish that calcium occupies $35 \%$ of the CTC in the $0-0.20 \mathrm{~m}$ layer and $30 \%$ of the CTCe in the $0.20-0.40 \mathrm{~m}$ layer, T4 - standard treatment, without application of limestone and agricultural gypsum) and four replicates. The parameters evaluated in the soybean crop were: height of insertion of the first pod, height of plants, final stand $\mathrm{ha}^{-1}$, mass of one hundred grains and grain productivity ha ${ }^{-1}$. The data were submitted to the $\mathrm{F}$ test and the means were compared by the Tukey test $(\mathrm{p}<0.05)$. It was verified that the treatment T2 presented higher height of insertion of the first pod than the standard treatment. For the other evaluated agronomic characteristics, there was no difference between treatments.
\end{abstract}

Keywords: Glycine $\max$ (L.) Merrill. Correctives. Agronomic characteristics.

\footnotetext{
${ }^{1}$ Pesquisador Científico, Dr. - IAC - CAP de Seringueira e Sistemas Agroflorestais, Votuporanga, SP;

2 Assistente Agropecuário - CDRS - DSMM/NPS, Fernandópolis, SP;

${ }^{3}$ Assistente Agropecuário - CDRS - DSMM/NPS, Araçatuba, SP;

${ }^{4}$ Doutoranda, MSc. - UNESP - Faculdade de Engenharia, Ilha Solteira, SP. Bolsista FAPESP;

${ }^{5}$ Professor Adjunto, Dr. - UNESP - Faculdade de Engenharia, Ilha Solteira, SP;

${ }^{6}$ Assistente Agropecuário - CDRS - CA Américo de Campos, Américo de Campos, SP.
} 


\section{INTRODUÇÃO}

Sistemas de manejo conservacionistas que têm o intuito de evitar o desgaste ou a degradação do solo vêm sendo difundidos na agricultura. Entre esses sistemas está o sistema de semeadura direta.

Neste sistema, geralmente, a correção da acidez do solo é realizada mediante aplicação de calcário na superfície, sem incorporação e, a baixa mobilidade dos produtos de dissolução do calcário aplicado na superfície limita sua eficiência na redução da acidez em camadas sub superficiais de solos com cargas variáveis e, que dependem da lixiviação de sais, orgânicos e, ou, inorgânicos, através do perfil do solo (CAIRES et al., 2006).

A calagem na superfície cria uma frente de correção da acidez do solo em profundidade, proporcional à dose e ao tempo (CAIRES et al., 2000; RHEINHEIMER et al., 2000), no entanto, vários trabalhos mostraram efeitos positivos da calagem superficial mais pronunciados nas camadas superficiais do solo (CAIRES et al., 1998, 1999; PÖTTKER; BEN, 1998; RHEINHEIMER et al., 2000; MOREIRA et al., 2001; PETRERE; ANGHINONI, 2001; CIOTTA et al., 2002) e, as limitações causadas pela acidez no subsolo à produtividade agrícola, devido à restrição ao crescimento radicular e à absorção de água e nutrientes pelas culturas, têm sido amplamente relatadas na literatura (PAVAN; BINGHAM; PRATT, 1982; RITCHEY; SILVA; COSTA, 1982; QUAGGIO, 2000).

A melhoria das condições do solo abaixo das camadas superficiais pode ser um fator de aumento e/ou estabilidade de produtividade das culturas, especialmente quando há ocorrência de veranicos, comuns nas regiões com inverno seco, notadamente no Cerrado, que apresentam deficiência de Ca na sub superfície do solo, associada ou não à toxidez de $\mathrm{Al}$ (COSTA, 2015).

Uma opção interessante para melhoria das camadas sub superficiais em sistemas de semeadura direta é a utilização do gesso agrícola, o qual tem sido utilizado em solos ácidos como um produto complementar ao calcário (BRAGA; VALE; MUNIZ, 1995; SILVA et al., 1998).

O presente trabalho foi realizado com o objetivo de avaliar o efeito da calagem e gessagem sobre a cultura da soja cultivada em sistema de semeadura direta na região Noroeste Paulista.

\section{MATERIAL E MÉTODO}

O experimento foi instalado no Centro Avançado de Pesquisa de Seringueira e Sistemas Agroflorestais, do Instituto Agronômico (IAC), da Agência Paulista de Tecnologia dos Agronegócios APTA/SAA, localizado no município de Votuporanga, SP, (20²0'S, 4958'W e $510 \mathrm{~m}$ de altitude), em um Argissolo Vermelho-Amarelo eutrófico (segundo o SiBCS, SANTOS et al., 2013).

O clima é o tropical com invernos secos (Aw na classificação de Köppen) com temperatura média anual de $24^{\circ} \mathrm{C}$, tendo a média das máximas de $31,2^{\circ} \mathrm{C}$ e a média das mínimas de $17,4^{\circ} \mathrm{C}$, enquanto que a precipitação pluvial média anual é de 1328,6 mm.

A área do experimento era destinada à produção de grãos, com sistema convencional de preparo do solo. Na safra 2008/09 a área foi cultivada com amendoim e sorgo granífero sobre a resteva do amendoim. Após o cultivo do sorgo, todas as semeaduras foram realizadas em sistema de semeadura direta. O sistema de rotação de culturas adotado no sistema, no período de novembro de 2009 a outubro de 2018, consta na Tabela 1. O sorgo forrageiro, a Urochloa ruziziensis e a U. brizantha foram utilizados como plantas de cobertura e não foram retirados da área. A quantidade de nutrientes utilizada no sistema, no período de novembro de 2009 a outubro de 2018, encontra-se na Tabela 2. 
Tabela 1. Rotação de cultura utilizada no período de novembro (Nov) de 2009 a outubro (Out) de 2018.

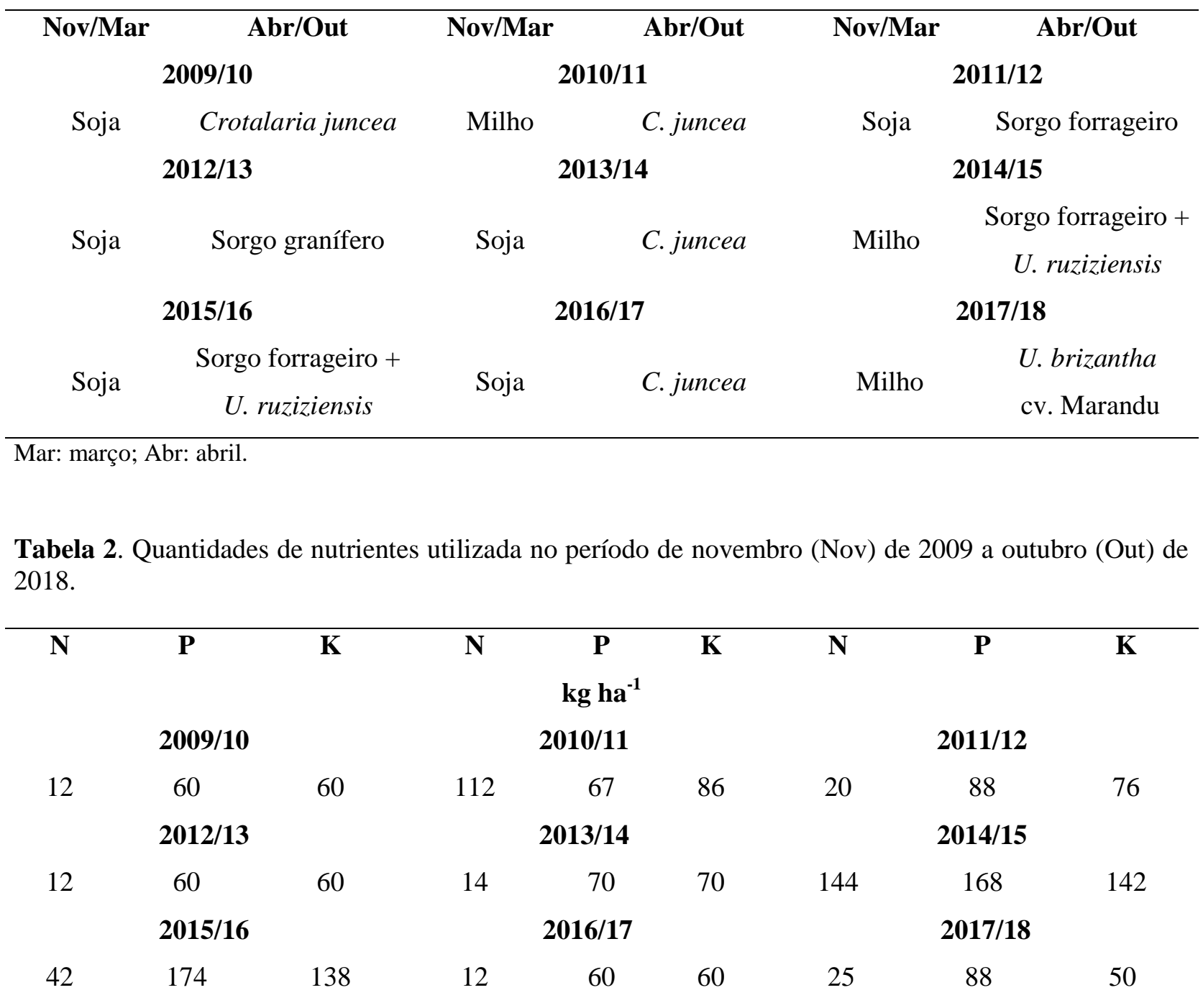

Foi realizada uma coleta de solo no dia 16/04/2018 para análise química de solo e determinação da sua fertilidade (RAIJ et al., 2001), nas camadas de 0-0,20 e 0,20-0,40 m de profundidade, e os resultados estão apresentados na Tabela 3.

Tabela 3. Valores referentes à análise de amostra de solo, nas camadas de 0-0,20 e 0,20-0,40 m, 2018.

\begin{tabular}{|c|c|c|c|c|c|c|c|c|c|c|}
\hline \multirow{2}{*}{ Camadas } & $\mathbf{P}$ & ${\mathrm{S}-\mathrm{SO}_{4}}_{4}$ & MO & pH & $\mathbf{K}$ & $\mathbf{C a}$ & $\mathbf{M g}$ & $\mathbf{H}+\mathrm{Al}$ & Al & $\mathbf{V}$ \\
\hline & \multicolumn{2}{|c|}{$\mathbf{m g ~ d m}{ }^{-3}$} & $\mathrm{~g} \mathrm{dm}^{-3}$ & & ---. & -- & lolc d & & -- & $\%$ \\
\hline $0-0,20$ & 15 & 10 & 14 & 4,4 & 0,9 & 16 & 5 & 29 & 3 & 43 \\
\hline $0,20-0,40$ & 10 & 26 & 13 & 4,0 & 1,6 & 10 & 4 & 36 & 6 & 31 \\
\hline
\end{tabular}

O delineamento experimental utilizado foi o em blocos casualizados com quatro repetições, utilizando-se quatro tratamentos: T1 - aplicação de calcário e gesso agrícola em superfície para estabelecer que, respectivamente, o cálcio ocupe $70 \%$ da capacidade de troca de cátions (CTC) na camada de 0-0,20 m e $60 \%$ da capacidade de troca de cátions efetiva (CTCe) na camada de 0,20-0,40 m; T2 - aplicação de calcário e gesso agrícola em superfície para estabelecer que o cálcio ocupe 52,5\% da CTC na camada de 0-0,20 m e 45\% da CTCe na camada de 0,20-0,40 m; T3 - aplicação de calcário e gesso agrícola em 
superfície para estabelecer que o cálcio ocupe 35\% da CTC na camada de 0-0,20 m e 30\% da CTCe na camada de 0,20-0,40 m; T4 - tratamento padrão (sem aplicação de calcário e gesso agrícola).

Cada parcela foi composta por dez linhas de soja (espaçamento de $0,50 \mathrm{~m}$ ) e $5 \mathrm{~m}$ de comprimento, totalizando $25 \mathrm{~m}^{2}$.

O calcário e o gesso agrícola foram aplicados manualmente sobre a superfície do solo, no dia $30 / 08 / 2018$.

No dia 27/11/2018 foi realizada uma roçada da $U$. brizantha presente na área para facilitar a semeadura da soja, pois a mesma apresentava, em média, $18840 \mathrm{~kg} \mathrm{ha}^{-1}$ de matéria verde.

A semeadura da soja foi realizada mecanicamente no sistema de semeadura direta sobre a palhada da $U$. brizantha no dia 24/11/2017, utilizando a cultivar 74HO112TP IPRO Paranaiba no espaçamento de $0,50 \mathrm{~m}$ e população de 280000 plantas ha ${ }^{-1}$, com adubação de base na dose de $300 \mathrm{~kg} \mathrm{ha}^{-1}$ do formulado 04-20-20.

Os parâmetros avaliados na cultura da soja foram: altura de inserção da primeira vagem, altura de plantas, estande final ha ${ }^{-1}$, massa de cem grãos e produtividade de grãos ha ${ }^{-1}$.

As avaliações foram realizadas no momento da colheita da cultura da soja, realizada no dia 01/04/2019. A massa de cem grãos e produtividade de grãos foi obtida padronizando-se a umidade dos grãos para $13 \%$ (base úmida).

A amostragem da altura de inserção da primeira vagem e altura de plantas foi realizada em cinco plantas de cada parcela, e a amostragem do estande final ha ${ }^{-1}$, massa de cem grãos e produtividade de grãos foi realizada em $3 \mathrm{~m}$ de duas linhas centrais de cada parcela.

As vagens foram debulhadas em debulhadora mecânica. Após a debulha os grãos foram pesados e mensurada sua umidade para o cálculo da produtividade de grãos. Em seguida separou-se cem grãos para cálculo da massa de cem grãos.

Os dados foram submetidos ao teste $\mathrm{F}$ e as médias foram comparadas pelo teste de Tukey ( $\mathrm{p}<0,05)$, com o uso do programa computacional Assistat (SILVA; AZEVEDO, 2016).

Os dados mensais de evapotranspiração potencial, precipitação pluvial e temperatura média de Votuporanga, SP, no período de novembro de 2018 a abril de 2019, encontram-se na Figura 1.

Figura 1. Dados de evapotranspiração potencial (ETP), precipitação pluvial (PP) e temperatura média (T), em Votuporanga, SP, no período estudado, novembro de 2018 a abril de 2019. Fonte: CIIAGRO (2019).

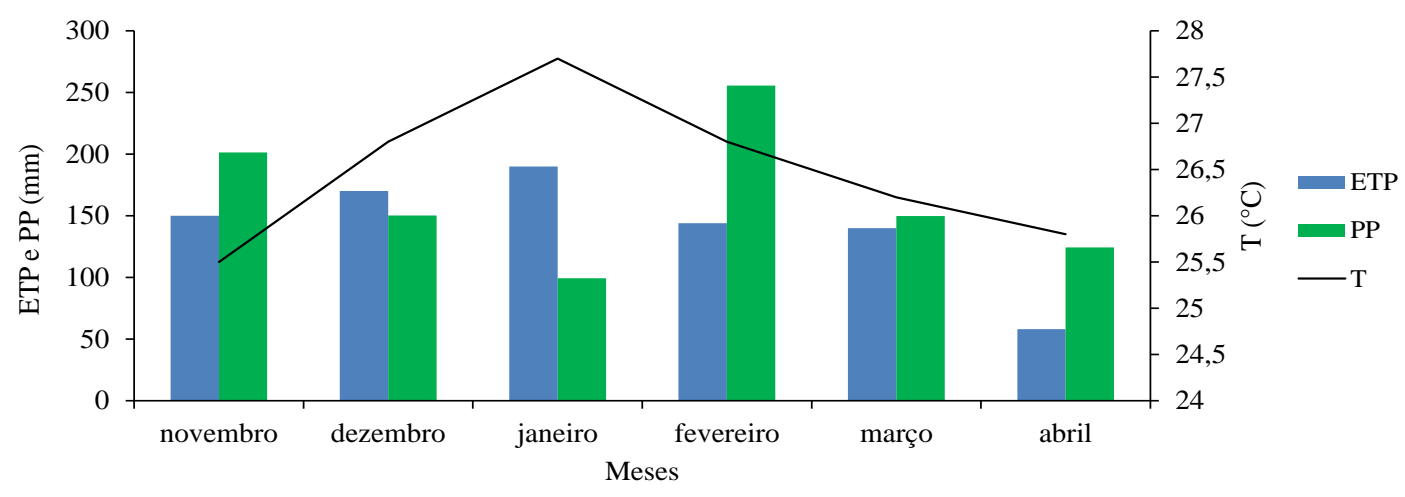

\section{RESULTADO E DISCUSSÃO}

As características agronômicas da cultura da soja estão demonstradas na Tabela 4. O tratamento T1 (aplicação de calcário e gesso agrícola em superfície para estabelecer que, respectivamente, o cálcio 
ocupe 70\% da CTC na camada de 0-0,20 m e 60\% da CTCe na camada de 0,20-0,40 m) apresentou menor massa de cem grãos e diferiu $(\mathrm{p}<0,05)$ do tratamento padrão (sem aplicação de calcário e gesso agrícola).

Tabela 4. Características agronômicas da cultura da soja, Votuporanga, SP, 2019.

\begin{tabular}{lccccc}
\hline Tratamentos $^{(\mathbf{1})}$ & $\begin{array}{c}\text { Altura de } \\
\text { inserção }^{(\mathbf{4})}\end{array}$ & $\begin{array}{c}\text { Altura de } \\
\text { plantas }\end{array}$ & $\begin{array}{c}\text { Estande final } \\
(\mathbf{m})\end{array}$ & $\begin{array}{c}\text { Massa de cem } \\
\text { grãos }\end{array}$ & $\begin{array}{c}\text { Produtividade } \\
\text { de grãos }\end{array}$ \\
T1 & $0,05^{(\mathrm{ns})}$ & 0,79 & 132500 & $13,03 \mathbf{b}^{(5)}$ & 4100 \\
T2 & 0,06 & 0,84 & 117778 & $13,57 \mathrm{ab}$ & 3514 \\
T3 & 0,04 & 0,76 & 108889 & $13,18 \mathrm{ab}$ & 4190 \\
T4 - Padrão & 0,04 & 0,78 & 139167 & $14,27 \mathrm{a}$ & 4735 \\
DMS $^{(2)}$ & 0,03 & 0,16 & 50723 & 1,17 & 1514 \\
CV $^{(3)}$ & 30,22 & 9,33 & 18,42 & 3,92 & 16,57 \\
\hline
\end{tabular}

(1) T1: 70\% da CTC (0-0,20 m) e 60\% da CTCe $(0,20-0,40 \mathrm{~m})$; T2: 52,5\% da CTC $(0-0,20 \mathrm{~m})$ e $45 \%$ da CTCe $(0,20-$ 0,40 m); T3: $35 \%$ da CTC $(0-0,20 \mathrm{~m})$ e $30 \%$ da CTCe $(0,20-0,40 \mathrm{~m})$; ${ }^{(2)}$ DMS: Diferença mínima significativa; ${ }^{(3)}$ CV: Coeficiente de variação; ${ }^{(4)}$ Altura de inserção: altura de inserção da primeira vagem; ${ }^{\left({ }^{(n)}\right)}$ : não-significativo; ${ }^{(5)}$ significativo a $5 \%$ de probabilidade.

Para as características altura de inserção da primeira vagem, altura de plantas, estande final ha $^{-1} \mathrm{e}$ produtividade de grãos $\mathrm{ha}^{-1}$ não houve diferença $(\mathrm{p}<0,05)$ entre os tratamentos. Caires et al. (2003) também não verificaram diferença em relação à produtividade de grãos de soja entre o tratamento padrão (sem aplicação de calcário e gesso) e os tratamentos com calagem e gessagem, em sistema de semeadura direta, em três anos de cultivo da soja. A ausência de resposta da soja à aplicação de gesso pode estar relacionada ao fato do crescimento do sistema radicular da soja, na ausência de déficit hídrico, não ser influenciado pela redução da saturação por $\mathrm{Al}$ no subsolo (Caires et al., 2001) e, a falta de resposta da soja à aplicação de calcário pode ser devido ao calcário ter sido aplicado na superfície do solo, tendo sua reação reduzida pelo menor contato entre as partículas de solo e corretivo, retardando, consequentemente, sua ação em profundidade no perfil (Ciotta et al., 2002).

\section{CONCLUSÃO}

A aplicação de calcário e gesso agrícola em superfície para estabelecer que, respectivamente, o cálcio ocupe $70 \%$ da CTC na camada de $0-0,20 \mathrm{~m}$ e $60 \%$ da CTCe na camada de 0,20-0,40 m, proporcionou menor altura de inserção da primeira vagem da cultura da soja, em sistema de semeadura direta, no Noroeste Paulista.

\section{AGRADECIMENTOS}

A todos os funcionários do Centro Avançado de Pesquisa de Seringueira e Sistemas Agroflorestais, do Instituto Agronômico - IAC, pelo apoio na instalação e condução do experimento. 


\section{REFERÊNCIAS}

CAIRES, E. F.; BANZATTO, D. A.; FONSECA, A. F. Calagem na superfície em sistema plantio direto. Revista Brasileira de Ciência do Solo, v. 24, n. 1, p. 161-169, 2000. Disponível em: http://www.scielo.br/pdf/rbcs/v24n1/18.pdf

CAIRES, E. F.; BARTH, G.; GARBUIO, F. J. Lime application in the establishment of a no-till system for grain crop production in Southern Brazil. Soil and Tillage Research, v. 89, n. 1, p. 3-12, 2006. Disponível em: https://doi.org/10.1016/j.still.2005.06.006

CAIRES, E. F.et al. Alterações químicas do solo e resposta da soja ao calcário e gesso aplicados na implantação do sistema plantio direto. Revista Brasileira de Ciência do Solo, v. 27, n. 2, p. 275-286, 2003. Disponível em: http://dx.doi.org/10.1590/S0100-06832003000200008

CAIRES, E. F.et al. Alterações de características químicas do solo e resposta da soja ao calcário e gesso aplicados na superfície em sistema de cultivo sem preparo de solo. Revista Brasileira de Ciência do Solo, v. 22, p. 27-34, 1998. Disponível em: http://dx.doi.org/10.1590/S0100-06831998000100004

CAIRES, E. F.et al. Crescimento radicular e nutrição da soja cultivada no sistema plantio direto em resposta ao calcário e gesso na superfície. Revista Brasileira de Ciência do Solo, v. 25, n. 4, p. 10291040, 2001. Disponível em: http://dx.doi.org/10.1590/S0100-06832001000400025

CAIRES, E. F.et al. Produção de milho, trigo e soja em função das alterações das características químicas do solo pela aplicação de calcário e gesso na superfície, em sistema plantio direto. Revista Brasileira de Ciência do Solo, v. 23, n. 1, p. 315-327, 1999. Disponível em: http://dx.doi.org/10.1590/S0100-06831999000200016

CENTRO INTEGRADO DE INFORMAÇÕES AGROMETEOROLÓGICAS - CIIAGRO. Resenha: Votuporanga no período de 01/11/2018 até 30/04/2019. São Paulo, 2019. Disponível em: http://www.ciiagro.sp.gov.br/ciiagroonline/Listagens/Resenha/LResenhaLocal.asp

CIOTTA, M. N. et al. Acidificação de um Latossolo sob plantio direto. Revista Brasileira de Ciência do Solo, v. 26, n. 4, p. 1055-1064, 2002. Disponível em: http://dx.doi.org/10.1590/S0100-06832002000400023

COSTA, C. H. M. Calagem superficial e aplicação de gesso em sistema plantio direto de longa duração: efeitos no solo e na sucessão milho/crambe/feijão-caupi. 2015. 97 f. (Tese de Doutorado) Faculdade de Ciências Agronômicas, Universidade Estadual Paulista, 2015.

MOREIRA, S. G.et al. Calagem em sistema de semeadura direta e efeitos sobre a acidez do solo, disponibilidade de nutrientes e produtividade de milho e soja. Revista Brasileira de Ciência do Solo, v. 25, p. 71-81, 2001. Disponível em: http://dx.doi.org/10.1590/S0100-06832001000100008

PAVAN, M. A.; BINGHAM, F. T.; PRATT, P. F. Toxicity of aluminum to coffee in Ultisols and Oxisols amended with $\mathrm{CaCO}_{3}, \mathrm{MgCO}_{3}$, and $\mathrm{CaSO}_{4} \cdot 2 \mathrm{H}_{2} \mathrm{O}$. Soil Science Society of America Journal, v. 46, n. 6, p. 1201-1207, 1982. Disponível em:

https://dl.sciencesocieties.org/publications/sssaj/abstracts/46/6/SS0460061201

PETRERE, C.; ANGHINONI, I. Alterações de atributos químicos no perfil do solo pela calagem superficial em campo nativo. Revista Brasileira de Ciência do Solo, v. 25, p. 885-895, 2001. Disponível em: http://dx.doi.org/10.1590/S0100-06832001000400011

PÖTTKER, D.; BEN, J. R. Calagem para uma rotação de culturas no sistema plantio direto. Revista Brasileira Ciência Solo, v. 22, n. 4, p. 675-684, 1998. Disponível em:

http://dx.doi.org/10.1590/S0100-06831998000400013 
RAIJ, B. van. et al.Análise química para avaliação da fertilidade do solo. Campinas: Instituto Agronômico; 2001.

RHEINHEIMER, D. S. et al. Alterações de atributos do solo pela calagem superficial e incorporada a partir de pastagem natural. Revista Brasileira de Ciência do Solo, v. 24, p. 797-805, 2000. Disponível em: http://www.scielo.br/pdf/rbcs/v24n4/12.pdf

RITCHEY, K. D.; SILVA, S. E.; COSTA, V. F. No TitCalcium deficiency in clayey B horizons of savannah Oxisolsle. Soil Science, v. 133, p. 378-382, 1982.

SANTOS, H. G.et al. Centro Nacional de Pesquisa de Solos. Sistema brasileiro de classificação de solos. 3. ed. Rio de Janeiro, 2013. 353 p.

SILVA, F. A. S.; AZEVEDO, C. A. V. The Assistat Software Version 7.7 and its use in the analysis of experimental data. African Journal of Agricultural Research, v. 11, n. 39, p. 3733-3740, 2016.

Disponível em: http://www.academicjournals.org/journal/AJAR/article-abstract/5E8596460818 